\title{
NEAR-OPTIMAL SPECTRUM ALLOCATION IN MULTI-TIER CELLULAR NETWORKS WITH RANDOM INELASTIC TRAFFIC
}

\author{
Wei Bao and Ben Liang
}

\author{
Department of Electrical and Computer Engineering, University of Toronto, Canada
}

\begin{abstract}
We present a new method for spectrum allocation in a heterogeneous cellular network with multiple tiers of randomly placed base stations and random user session arrivals. Different from previous works, inelastic network traffic is considered, so as to accommodate application sessions with fixed data rate requirements. We first quantify the average downlink sum throughput of the network in terms of a given spectrum allocation vector. We then derive concave upper and lower bounds to the throughput to allow efficient approximate solutions to optimize spectrum allocation. We show that the proposed approach has a worst case optimization performance gap of $12.6 \%$ and further demonstrate via simulation that its actual performance is often near optimal.
\end{abstract}

\section{INTRODUCTION}

Driven by recent advances in sophisticated mobile phones and devices, multimedia applications have proliferated in mobile networks. A defining characteristic of multimedia traffic is inelasticity. The required network throughput for a multimedia session is defined in discrete levels, and the minimum throughput must be satisfied, or the session is rejected or dropped. The stringent requirements of inelastic traffic brings substantial challenges to resource management in wireless networks.

Further complicating the resource management problem, the multi-tier cellular structure, with microcells, femtocells, and other small cells, is expected to be widely adopted to improve throughput and coverage. The radio spectrum licensed by a network operator must be shared by base stations (BSs) of widely different power and coverage areas. Furthermore, small cells are usually deployed randomly, creating complicated cell shapes and interference patterns. In order to avoid cross-tier interference, and the prohibitive complexity in tracking and provisioning for such interference, it is commonly advocated to allocate non-overlapping portions of the spectrum to different tiers of BSs. Early works [1,2] consider a fixed set of BSs and User Equipments (UEs) without accounting for their random spatial patterns.

More recent works on the spectrum allocation problem utilize a stochastic geometric approach $[3,4,5,6]$. Limited to two

This work has been funded in part by Bell Canada and the Natural Sciences and Engineering Research Council of Canada. tiers of BSs, optimal spectrum allocation has been derived in $[7,8]$. Joint spectrum allocation and user association have been studied in [9] for two tiers of BSs, and in [10] for general multiple tiers of BSs. However, all of these works assume that all UEs are accepted by the system, so that they cannot accommodate the aforementioned scenario where inelastic traffic maybe rejected.

In this work, we are interested in studying the optimization of spectrum allocation in a multi-tier cellular network with inelastic traffic, in order to maximize the average downlink sum throughput. Different from previous works, we assume that if the inelastic traffic load has reached the allowed capacity at a $\mathrm{BS}$, no new UEs will be accepted. This user rejection problem in multi-cell networks has been well studied in the existing literature, without considering the randomness in cell number or location, e.g., $[11,12]$. Instead, we model the random spatial patterns of BSs and UEs as Poisson point processes. Our work also builds on recent downlink performance analysis based on stochastic geometry, e.g., $[13,14]$ for the single-tier case and $[15,16]$ for the multi-tier case. However, none of them consider spectrum allocation among BSs. To our best knowledge, this is the first work to study multi-tier spectrum allocation with inelastic traffic.

The hard limit of inelasticity imposes substantial challenges to the analysis and optimization of system performance. Furthermore, the standard stochastic geometric approaches often lead to non-closed form expressions that require difficult numerical computation. In this work, we first quantify the average sum throughput in terms of a given spectrum allocation, taking into consideration the probability of UE rejection. Then, we develop concave upper and lower bounds to provide efficient approximate solutions to the throughput optimization problem. We further show that the proposed approach has a worst case optimization performance gap within $12.6 \%$ and demonstrate via simulation that its actual performance is often near optimal.

\section{SYSTEM MODEL AND OBJECTIVE}

\subsection{Multi-tier Cellular Network}

We consider a heterogeneous cellular network with randomly spatially distributed $K$ tiers of BSs. As in conventional stochastic geometric modeling of multi-tier cellular networks $[9,15,17,18,19]$, each tier of BSs is assumed to indepen- 


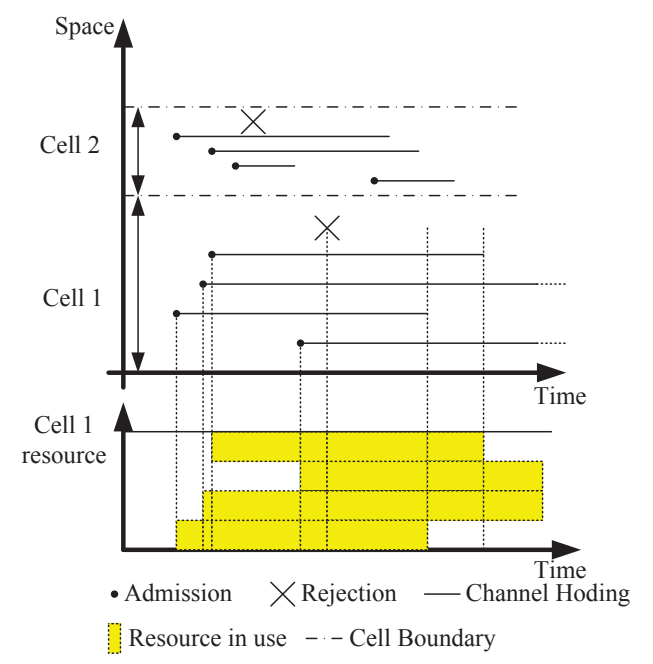

Fig. 1. System model, with $N_{1}=4$.

dently form a homogeneous Poisson point process (PPP) in two-dimensional Euclidean space $\mathbb{R}^{2}$. Let $\Phi_{k}$ denote the PP$\mathrm{P}$ corresponding to tier- $k \mathrm{BSs}$, with intensity $\lambda_{k}$ units $/ \mathrm{m}^{2}$. The locations of BSs do not change over time.

UEs are located randomly in the same two-dimensional space as the BSs, as well as in time. The arrival of UEs are modeled as a homogeneous PPP $\Psi$ with intensity $\mu$ units $/\left(\mathrm{m}^{2} \cdot \mathrm{min}\right)$ in $\mathbb{R}^{3}$. If a UE is admitted by the system, it will stay in the system for a duration that is exponentially distributed with mean $\frac{1}{\nu}$ minutes. We assume that a UE remains connected with the same BS when it is in an active session. When a newly arriving UE is rejected (see details in Section 2.3), it leaves the system immediately.

\subsection{Power and Pathloss}

We define the tiers of BSs by their transmission powers. Let $P_{k}$ be the transmission power of tier- $k$ BSs, which is a given parameter. If $P_{t}(\mathbf{x})$ is the transmission power from a BS at location $\mathbf{x}$ and $P_{r}(\mathbf{y})$ is the received power at $\mathbf{y}$, we assume $P_{r}(\mathbf{y})=\frac{P_{t}(\mathbf{x}) h_{\mathbf{x}, \mathbf{y}}}{\beta|\mathbf{x}-\mathbf{y}|^{\gamma}}$, where $\beta|\mathbf{x}-\mathbf{y}|^{\gamma}$ is the propagation loss function with predetermined constants $\beta$ and $\gamma$, and $h_{\mathbf{x}, \mathbf{y}}$ is the fast fading term. Corresponding to common Rayleigh fading with power normalization, $h_{\mathbf{x}, \mathbf{y}}$ is independently exponentially distributed with unit mean.

We assume each BS is connected to the core network by separate high-capacity wired or wireless links that have no influence on our performance analysis. In addition, because we focus on downlink analysis, we assume that the downlink and uplink of the system are operated in different spectrum, so that the uplink interference and capacity have no influence on the downlink analysis.

\subsection{Spectrum Allocation and UE Rejection}

Different tiers of BSs are allocated separated spectrum. Given the total downlink spectrum bandwidth $W$, the network opera- tor allocates $\eta_{k} W$ to each tier- $k \mathrm{BS}$, where $\eta_{k}$ are the spectrum allocation factors and $\sum_{k=1}^{K} \eta_{k}=1$. Let $\boldsymbol{\eta}=\left(\eta_{1}, \eta_{2}, \ldots, \eta_{K}\right)$. Note that BSs in the same tier are operated on the same spectrum and will interfere at the UEs.

Each UE requests a connection with the BS that provides the maximum received power. ${ }^{1}$ Then, the resultant cell splitting forms a generalized Dirichlet tessellation, or weighted Poisson Voronoi [20]. We assume that each admitted UE requires a downlink frequency bandwidth of 1 (i.e., unit frequency bandwidth). Therefore, a tier- $k$ BS can accommodate at most $N_{k}=$ $\eta_{k} W$ UEs. If there are already $N_{k}$ UEs connecting to it, new UE arrivals are rejected. Otherwise, a newly arriving UE is randomly allocated an available unit frequency bandwidth. Figure 1 illustrates the system model with an example of UE rejection. We assume that $N_{k}$ is a large number ${ }^{2}$.

\subsection{Coverage Probability and UE Data Rate}

Let $T$ denote the minimum required Signal-to-Interference Ratio (SIR) of UEs. The coverage probability of a UE, $P_{\text {cover }}$, is defined as the probability that its SIR is no less than $T$ [3]. The thermal noise is assumed to be negligible compared with interference. Then, a UE's data rate is $\log (1+T)$ if the SIR is no less than $T$, and its data rate is 0 if the SIR is less than $T$ (i.e., outage occurs). Thus, the overall data rate of the UE is $\log (1+T) P_{\text {cover }}$.

In this work, we aim to optimize the spectrum allocation factors $\boldsymbol{\eta}$, in order to maximize the the long-term average downlink sum throughput over all UEs, which equals the expected downlink sum rate at any random snapshot.

\section{DOWNLINK THROUGHPUT DERIVATION}

\subsection{Interference Analysis and Coverage Probability}

Consider a reference UE, termed the typical UE, communicating with its BS, termed the typical BS. The average UE coverage probability in the system is the same as the coverage probability of the typical UE [3]. Furthermore, due to the stationarity of UEs and BSs, we can re-define the coordinates so that the typical UE is located at $\mathbf{0}$.

First, we derive the coverage probability given that the typical UE is at distance $d$ from its associated tier- $k$ BS. In this case, the overall interference, $I_{k}(d)$, to the typical UE is the sum interference from all other tier- $k$ BSs that are actively using the same unit frequency band as the typical UE.

Let $\Phi_{k}^{\prime}$ be the Palm point process corresponding to all tier$k$ BSs other than the typical BS using the same unit frequency band as the typical UE, given that the typical BS is located

\footnotetext{
${ }^{1}$ This model can be easily extended to accommodate the flexible user association scheme [10,17, 18,19], in which each tier of BSs is assigned with an association bias value, and a UE is associated with a BS providing the largest biased received power.

${ }^{2}$ Even though we assume that $N_{k}$ is large, it does not imply that all UEs will be admitted, as the traffic load (average UE per BS) may increase in the same scale of $N_{k}$.
} 
at distance $d$ from the typical UE. By standard Palm theory, $\Phi_{k}^{\prime}$ is a PPP with intensity 0 in $\mathcal{B}(\mathbf{0}, d)$ and intensity $p_{k} \lambda_{k}$ in $\mathbb{R}^{2} \backslash \mathcal{B}(\mathbf{0}, d)$, where $\mathcal{B}(\mathbf{0}, d)$ denotes the disk region centered at $\mathbf{0}$ with radius $d$, and $p_{k}$ is the probability that the unit frequency band of the typical UE is in use at a non-typical tier- $k$ BS.

Then, $I_{k}(d)=\sum_{\mathbf{x} \in \Phi_{k}^{\prime}} \frac{P_{k} h_{\mathbf{x}, 0}}{\beta|\mathbf{x}|^{\gamma}}$, and its distribution is derived through its Laplace transform as follows:

$$
\begin{aligned}
& \mathcal{L}_{I_{k}}(d, s)=\mathbf{E}\left[\exp \left(-\sum_{\mathbf{x} \in \Phi_{k}^{\prime}} \frac{s P_{k} h_{\mathbf{x}, \mathbf{0}}}{\beta|\mathbf{x}|^{\gamma}}\right)\right] \\
= & \exp \left(-2 \pi p_{k} \lambda_{k} \int_{d}^{\infty} \frac{\frac{s P_{k} r}{\beta}}{\frac{s P_{k}}{\beta}+r^{\gamma}} \mathrm{d} r\right),
\end{aligned}
$$

where the second equality is obtained from the Laplace functional of PPP $\Phi_{k}^{\prime}$.

Let $P_{\text {cover }, k}(d)$ denote the conditional coverage probability of the typical UE given $k$ and $d$. We have

$$
\begin{aligned}
P_{\text {cover }, k}(d) & =\mathbf{P}\left[\frac{P_{k} h_{\mathbf{x}_{B}, \mathbf{0}}}{\beta d^{\gamma}} \geq T I_{k}(d)\right]=\left.\mathcal{L}_{I_{k}}(d, s)\right|_{s=\frac{T \beta d^{\gamma}}{P_{k}}}, \\
& =\exp \left(-\pi p_{k} \lambda_{k} T^{\frac{2}{\gamma}} d^{2} \int_{\left(\frac{1}{T}\right)^{\frac{2}{\gamma}}}^{\infty} \frac{1}{1+t^{\frac{\gamma}{2}}} \mathrm{~d} t\right),
\end{aligned}
$$

where $\mathbf{x}_{B}$ is the coordinate of the typical BS and $\left|\mathbf{x}_{B}\right|=d$.

Furthermore, the probability density function of the distance between the typical UE and its associated tier- $k$ BS is known to be $g_{k}(d)=\frac{2 \pi \lambda_{k}}{A_{k}} d \exp \left(-\pi d^{2} \frac{\lambda_{k}}{A_{k}}\right)$ [17], where $A_{k}=$ $\frac{\lambda_{k}\left(P_{k}\right)^{\frac{2}{\gamma}}}{\sum_{j=1}^{K} \lambda_{j}\left(P_{j}\right)^{\frac{2}{\gamma}}}$ represents the proportion of area that tier- $k$ cells cover. Then, we can derive the coverage probability $P_{\text {cover }, k}$ of the typical UE connected to a tier- $k \mathrm{BS}$ :

$$
P_{\text {cover }, k}=\int_{0}^{\infty} g_{k}(d) P_{\text {cover }, k}(d) \mathrm{d} d=\frac{1}{1+C A_{k} p_{k}},
$$

where $C=(T)^{\frac{2}{\gamma}} \int_{\left(\frac{1}{T}\right)^{\frac{2}{\gamma}}}^{\infty} \frac{1}{1+t^{\gamma / 2}} \mathrm{~d} t$ is a system-level constant only related to $\gamma$ and $T$. The details are omitted for brevity.

\subsection{Normalized Downlink Throughput}

Let $M_{k}$ denote the number of UEs in a tier- $k$ cell. Its mean is denoted by $\mathbf{E}\left(M_{k}\right)$. Then the average throughput of a tier- $k$ BS is $^{3}$

$$
\bar{R}_{k}=\mathbf{E}\left(M_{k}\right) \log (1+T) P_{\text {cover }, k},
$$

and the average downlink sum throughput per unit area (normalized throughput) is

$$
\bar{R}=\sum_{k=1}^{K} \lambda_{k} \bar{R}_{k}=\sum_{k=1}^{K} \lambda_{k} \mathbf{E}\left(M_{k}\right) \frac{\log (1+T)}{1+C A_{k} p_{k}} .
$$

\footnotetext{
${ }^{3}$ We have used an approximation that the coverage event and $M_{k}$ are independent for mathematical tractability. In reality, their dependency is negligible, which has been verified by simulations in $[18,14]$. In Section 5 , we also show via simulation that the throughput maximization results based on this approximation is close to those without it.
}

Next, we aim to derive $p_{k}$ and $\mathbf{E}\left(M_{k}\right)$, which are required to compute $\bar{R}$.

First, the cell size of a tier- $k$ cell, $S_{k}$, is known to have the following probability density function $[21,18]$ :

$$
f_{S_{k}}(c)=\frac{3.5^{3.5}}{\Gamma(3.5)} \frac{\lambda_{k}}{A_{k}}\left(\frac{\lambda_{k}}{A_{k}} c\right)^{2.5} e^{-3.5 c \frac{\lambda_{k}}{A_{k}}}
$$

Given a tier- $k$ cell with $S_{k}=c$, and with $N_{k}$ bandwidth, the cell is equivalent to an $M / M / N_{k} / N_{k}$ queueing system with user arrival rate $\mu c$ and departure rate $\nu$. In this case, the probability that there are $n$ UEs in the cell is $P\left(M_{k}=n \mid S_{k}=c\right)=$ $\frac{\left(\frac{\mu c}{\nu}\right)^{n}}{n !} / \sum_{i=0}^{N_{k}} \frac{\left(\frac{\mu c}{\nu}\right)^{i}}{i !}$. Then, the probability that a unit bandwidth is in use is

$$
p_{k}(c)=\sum_{n=0}^{N_{k}} \frac{n}{N_{k}} \frac{\frac{\left(\frac{\mu c}{\nu}\right)^{n}}{n !}}{\sum_{i=0}^{N_{k}} \frac{\left(\frac{\mu c}{\nu}\right)^{i}}{i !}}= \begin{cases}\frac{\mu c}{\nu N_{k}} & \text { if } \frac{\mu c}{\nu N_{k}}<1 \\ 1 & \text { if } \frac{\mu c}{\nu N_{k}} \geq 1 .\end{cases}
$$

where the second equality holds since $N_{k}$ is a large number.

Hence, $p_{k}$ can be computed as

$$
\begin{aligned}
& p_{k}=p_{k}(c) f_{S_{k}}(c) \mathrm{d} c \\
= & \frac{1}{\alpha_{k}}-\frac{1}{\alpha_{k}} \frac{3.5^{3.5}}{\Gamma(3.5)} \int_{1}^{\infty}(y-1) \alpha_{k}^{4.5} y^{2.5} e^{-3.5 \alpha_{k} y} \mathrm{~d} y,
\end{aligned}
$$

where $\alpha_{k}=\frac{\lambda_{k} \nu N_{k}}{A_{k} \mu}$. For presentation convenience, we define $f\left(\alpha_{k}\right)=\frac{3.5^{3.5}}{\Gamma(3.5)} \int_{1}^{\infty}(y-1) \alpha_{k}^{4.5} y^{2.5} e^{-3.5 \alpha_{k} y} \mathrm{~d} y$.

Finally, similar manipulation leads to

$$
\mathbf{E}\left(M_{k}\right)=\frac{A_{k} \mu}{\lambda_{k} \nu}-\frac{A_{k} \mu}{\lambda_{k} \nu} f\left(\alpha_{k}\right)
$$

Substituting (7) and (8) into (4), we can compute the normalized system throughput.

\section{BOUNDING FOR SPECTRUM ALLOCATION}

\subsection{Optimization Problem Formulation}

We aim to maximize $\bar{R}$ with respect to $\boldsymbol{\eta}$. As there is a one-toone mapping between $\boldsymbol{\eta}$ and $\boldsymbol{\alpha}=\left(\alpha_{1}, \alpha_{2}, \ldots, \alpha_{K}\right)$, we instead study the optimization over $\boldsymbol{\alpha}$ for analytical convenience. This is formally stated as optimization problem $\mathbf{P}$ :

$$
\begin{array}{ll}
\underset{\boldsymbol{\alpha}}{\operatorname{maximize}} & \bar{R}(\boldsymbol{\alpha})=\sum_{k=1}^{K} \frac{A_{k}\left(1-f\left(\alpha_{k}\right)\right)}{1+A_{k} C \frac{1}{\alpha_{k}}\left(1-f\left(\alpha_{k}\right)\right)}, \\
\text { subject to } & \sum_{k=1}^{K} \frac{\alpha_{k} A_{k} \mu}{\lambda_{k} \nu}=W, \quad \alpha_{k} \geq 1, \forall k .
\end{array}
$$

Note that $1 / \alpha_{k}$ represents the average traffic load per tier-k unit frequency band. Therefore, we consider the constraint that $\alpha_{k} \geq 1$ in (10). $\log (1+T)$ and $\mu / \nu$ are omitted in (9) for presentation convenience.

Problem $\mathbf{P}$ is in non-closed form and is non-convex. Thus it cannot be solved using a standard method. Instead, we propose an efficient solution through bounding $f(\alpha)$ and $\bar{R}(\boldsymbol{\alpha})$. 


\subsection{Bounding the Optimization Problem}

As stated in the following lemma, we are able to derive closed form upper and lower bounds of $f(\alpha)$. The proof is omitted due to space limitation.

Lemma $1 f_{l b}(\alpha)<f(\alpha)<f_{u b}(\alpha)$ on $[1, \infty)$, where $f_{l b}(\alpha)=$ $\frac{3.5^{4}}{3 !} e^{-3.5 \alpha} \cdot\left[\frac{\alpha^{2}}{3.5^{2}}+\frac{4 \alpha}{3.5^{3}}+\frac{6}{3.5^{4}}\right]$ and $f_{u b}(\alpha)=\frac{3.5^{5}}{4 !} e^{-3.5 \alpha}$. $\left[\frac{\alpha^{3}}{3.5^{2}}+\frac{6 \alpha^{2}}{3.5^{3}}+\frac{18 \alpha}{3.5^{4}}+\frac{24}{3.5^{5}}\right]$.

As a consequence, we can define the upper and lower bounds of $\bar{R}(\boldsymbol{\alpha})$ as $\bar{R}_{u b}(\boldsymbol{\alpha})=\sum_{k=1}^{K} \frac{A_{k}\left(1-f_{l b}\left(\alpha_{k}\right)\right)}{1+A_{k} C \frac{1}{\alpha_{k}}\left(1-f_{l b}\left(\alpha_{k}\right)\right)}$ and $\bar{R}_{l b}(\boldsymbol{\alpha})=\sum_{k=1}^{K} \frac{A_{k}\left(1-f_{u b}\left(\alpha_{k}\right)\right)}{1+A_{k} C \frac{1}{\alpha_{k}}\left(1-f_{u b}\left(\alpha_{k}\right)\right)}$ respectively. Thus, instead of solving the original problem $\mathbf{P}$ directly, we propose to solve the following two problems separately:

$$
\begin{array}{llll}
\text { P1 } & \underset{\boldsymbol{\alpha}}{\operatorname{maximize}} & \bar{R}_{u b}(\boldsymbol{\alpha}) & \text { subject to (10), } \\
\text { P2 } & \underset{\boldsymbol{\alpha}}{\operatorname{maximize}} & \bar{R}_{l b}(\boldsymbol{\alpha}) & \text { subject to (10). }
\end{array}
$$

Note that both $\bar{R}_{u b}(\boldsymbol{\alpha})$ and $\bar{R}_{u b}(\boldsymbol{\alpha})$ are concave functions, and (10) is linear. Therefore, $\mathbf{P 1}$ and $\mathbf{P 2}$ can be efficiently solved by standard convex optimization methods.

\subsection{Performance Bound Analysis}

Let $\boldsymbol{\alpha}^{*}, \widehat{\boldsymbol{\alpha}}^{*}$, and $\widetilde{\boldsymbol{\alpha}}^{*}$ denote the optimal solution to problems $\mathbf{P}$, $\mathbf{P} 1$, and $\mathbf{P} 2$ respectively. We quantify $\Delta_{1}=\frac{\left|\bar{R}_{u b}\left(\widehat{\boldsymbol{\alpha}}^{*}\right)-\bar{R}\left(\boldsymbol{\alpha}^{*}\right)\right|}{\bar{R}\left(\boldsymbol{\alpha}^{*}\right)}$ and $\Delta_{2}=\frac{\left|\bar{R}_{l b}\left(\widetilde{\boldsymbol{\alpha}}^{*}\right)-\bar{R}\left(\boldsymbol{\alpha}^{*}\right)\right|}{\bar{R}\left(\boldsymbol{\alpha}^{*}\right)}$ as the relative performance gap corresponding to $\widehat{\boldsymbol{\alpha}}^{*}$ and $\widetilde{\boldsymbol{\alpha}}^{*}$ respectively.

Let $\Delta=\frac{\bar{R}_{u b}\left(\widehat{\boldsymbol{\alpha}}^{*}\right)-\bar{R}_{l b}\left(\widetilde{\boldsymbol{\alpha}}^{*}\right)}{\bar{R}\left(\boldsymbol{\alpha}^{*}\right)}$. Clearly, $\Delta \geq \max \left(\Delta_{1}, \Delta_{2}\right)$. Then, we can bound the worst case performance gap of the proposed solution as follows:

$$
\begin{aligned}
\Delta & =\frac{\bar{R}_{u b}\left(\widehat{\boldsymbol{\alpha}}^{*}\right)-\bar{R}_{l b}\left(\widehat{\boldsymbol{\alpha}}^{*}\right)}{\bar{R}\left(\boldsymbol{\alpha}^{*}\right)} \leq \frac{\bar{R}_{u b}\left(\widehat{\boldsymbol{\alpha}}^{*}\right)-\bar{R}_{l b}\left(\widehat{\boldsymbol{\alpha}}^{*}\right)}{\bar{R}_{l b}\left(\widehat{\boldsymbol{\alpha}}^{*}\right)} \\
& \leq \max _{k}\left[\frac{\frac{A_{k}\left(1-f_{l b}\left(\widehat{\alpha}_{k}^{*}\right)\right)}{1+A_{k} C \frac{1}{\widehat{\alpha}_{k}^{*}}\left(1-f_{l b}\left(\widehat{\alpha}_{k}^{*}\right)\right)}-\frac{A_{k}\left(1-f_{u b}\left(\widehat{\alpha}_{k}^{*}\right)\right)}{1+A_{k} C \frac{1}{\widehat{\alpha}_{k}^{*}}\left(1-f_{u b}\left(\widehat{\alpha}_{k}^{*}\right)\right)}}{\frac{A_{k}\left(1-f_{u b}\left(\widehat{\alpha}_{k}^{*}\right)\right)}{1+A_{k} C \frac{1}{\widehat{\alpha}_{k}^{*}}\left(1-f_{u b}\left(\widehat{\alpha}_{k}^{*}\right)\right)}}\right] \\
& \leq \max _{\alpha \in[1, \infty)} \frac{f_{u b}(\alpha)-f_{l b}(\alpha)}{1-f_{u b}(\alpha)}=12.6 \% .
\end{aligned}
$$

\section{NUMERICAL STUDY}

In addition to the above performance bound, we further evaluate the proposed solution via computer simulation. We first obtain optimal solutions to $\mathbf{P} 1$ and $\mathbf{P 2}$ as $\widehat{\boldsymbol{\eta}}^{*}$ and $\widetilde{\boldsymbol{\eta}}^{*}$, respectively. We further obtain $\boldsymbol{\eta}^{*}$ from exhaustive search. We then apply these spectrum allocation factors to a simulated network and compute the resultant throughput.

In Figs. 2 and 3, the following network parameters are used: $K=3 ;\left(\lambda_{1}, \lambda_{2}, \lambda_{3}\right)=(1,5,10)$ units $/ \mathrm{km}^{2} ;\left(P_{1}, P_{2}, P_{3}\right)=$

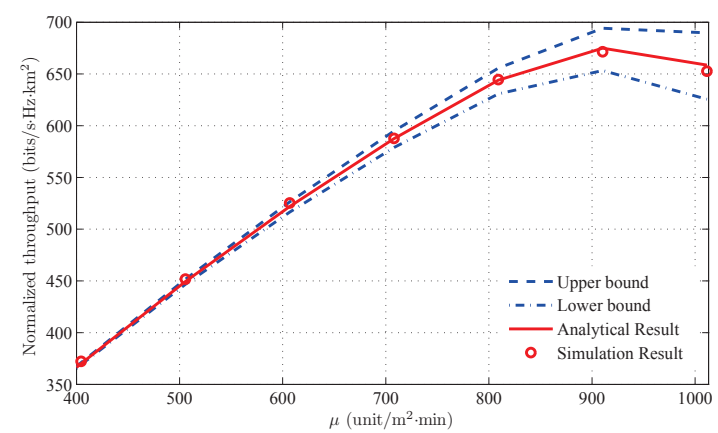

Fig. 2. Optimal normalized throughput under different $\mu$.

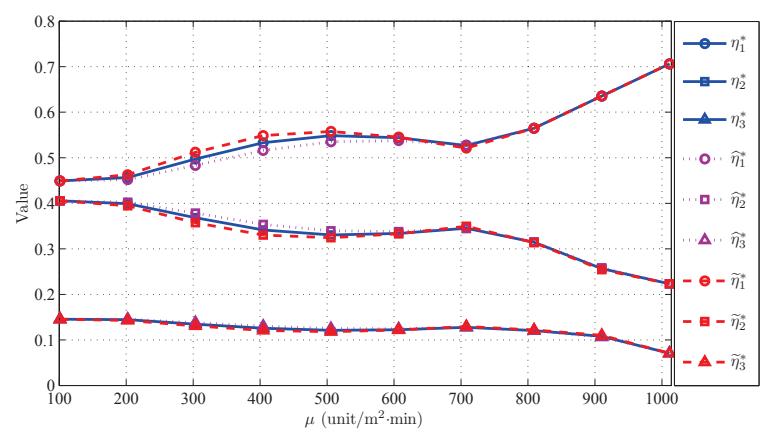

Fig. 3. Approximations of $\boldsymbol{\eta}^{*}$ under different $\mu$.

$(50,40,30) \mathrm{dBm} ; \nu=1$ units/min; $W=400$ units; $\gamma=4$; and $T=1$. Note that we choose the UE density (in space-time) to satisfy $\mu \leq 1011.4$ units $/ \mathrm{m}^{2}$. min such that the optimization problem is feasible. In each round of simulation, UEs and BSs are generated on a $10 \mathrm{~km} \times 10 \mathrm{~km}$ square, and the UEs in the central $5 \mathrm{~km} \times 5 \mathrm{~km}$ square are selected for performance evaluation in order to remove the edge effect. Each data point is averaged over 100 rounds of simulation.

Fig. 2 shows the normalized throughput bounds obtained from $\widehat{\boldsymbol{\eta}}^{*}$ (upper bound) and $\widetilde{\boldsymbol{\eta}}^{*}$ (lower bound), respectively. We also show the throughput under $\boldsymbol{\eta}^{*}$ for reference. Our numerical results demonstrate that the bounding approximations provide nearly optimal throughput for a wide range of $\mu$. Fig. 3 further shows $\widehat{\boldsymbol{\eta}}^{*}$ and $\widetilde{\boldsymbol{\eta}}^{*}$ versus $\mu$. We observe that both of them closely match $\eta^{*}$ for all $\mu$. These figures demonstrate that the proposed solution often performs much closer to optimality than the analytical bound derived in Section 4.3.

\section{CONCLUSION}

A new spectrum allocation method is presented for multi-tier wireless networks with inelastic traffic, with an aim to maximize the downlink sum throughput. The effect of spectrum allocation is derived through a stochastic geometric approach, with consideration for user rejection when a BS is at full capacity. The proposed concave upper and lower bounds are shown, both analytically and by simulation, to allow accurate approximation to the optimal solution. 


\section{REFERENCES}

[1] V. Chandrasekhar and J. Andrews, "Spectrum allocation in tiered cellular networks," IEEE Trans. on Communications, vol. 57, no. 10, pp. 3059-3068, Oct. 2009.

[2] I. Guvenc, M.-R. Jeong, F. Watanabe, and H. Inamura, "A hybrid frequency assignment for femtocells and coverage area analysis for co-channel operation," IEEE Communications Letters, vol. 12, no. 12, pp. 880-882, Dec. 2008.

[3] F. Baccelli and B. Blaszczyszyn, "Stochastic geometry and wireless networks, volume 1: Theory," Foundations and Trends in Networking, vol. 3, no. 3-4, pp. 249 - 449, 2009.

[4] - "Stochastic geometry and wireless networks, volume 2: Applications," Foundations and Trends in Networking, vol. 4, no. 1-2, pp. 1-312, 2009.

[5] M. Haenggi, J. G. Andrews, F. Baccelli, O. Dousse, and M. Franceschetti, "Stochastic geometry and random graphs for the analysis and design of wireless networks," IEEE Journal on Selected Areas in Communications, vol. 27, no. 7, pp. 1029 - 1046, Sep. 2009.

[6] D. Stoyan, W. Kendall, and J. Mecke, Stochastic Geometry and Its Applications, 2nd ed. Wiley, 1995.

[7] W. C. Cheung, T. Q. S. Quek, and M. Kountouris, "Throughput optimization, spectrum allocation, and access control in two-tier femtocell networks," IEEE Journal on Selected Areas in Communications, vol. 30, no. 3, pp. 561-574, Apr. 2012.

[8] _ , "Spectrum allocation and optimization in femtocell networks," in Proc. of IEEE ICC, Ottawa, Canada, Jun. 2012.

[9] S. Singh and J. G. Andrews, "Joint resource partitioning and offloading in heterogeneous cellular networks," arXiv:1303.7039 [cs.IT], Aug. 2013.

[10] W. Bao and B. Liang, "Structured spectrum allocation and user association in heterogeneous cellular networks," in Proc. of IEEE INFOCOM, Toronto, Canada, Apr. 2014.

[11] L. Cimini, G. Foschini, and C.-L. I, "Call blocking performance of distributed algorithms for dynamic channel allocation in microcells," in Proc. of IEEE ICC, Chicago, IL, Jun. 1992.

[12] S. Anand, A. Sridharan, and K. Sivarajan, "Performance analysis of channelized cellular systems with dynamic channel allocation," IEEE Trans. on Vehicular Technology, vol. 52, no. 4, pp. 847-859, Jul. 2003.

[13] J. G. Andrews, F. Baccelli, and R. Ganti, "A tractable approach to coverage and rate in cellular networks," IEEE Trans. on Communications, vol. 59, no. 11, pp. 31223134, Nov. 2011.
[14] S. M. Yu and S.-L. Kim, "Downlink capacity and base station density in cellular networks," in Proc. of IEEE International Symposium on Modeling and Optimization in Mobile, Ad Hoc, and Wireless Networks (WiOpt), Tsukuba Science City, Japan, May 2013.

[15] H. Dhillon, R. Ganti, F. Baccelli, and J. G. Andrews, "Modeling and analysis of K-tier downlink heterogeneous cellular networks," IEEE Journal on Selected Areas in Communications, vol. 30, no. 3, pp. 550-560, Apr. 2012.

[16] H.-S. Jo, P. Xia, and J. G. Andrews, "Open, closed, and shared access femtocells in the downlink," EURASIP Journal on Wireless Communications and Networking, Dec. 2012.

[17] H.-S. Jo, Y. J. Sang, P. Xia, and J. G. Andrews, "Heterogeneous cellular networks with flexible cell association: A comprehensive downlink SINR analysis," IEEE Trans. on Wireless Communications, vol. 11, no. 10, pp. 34843495, Oct. 2012.

[18] S. Singh, H. Dhillon, and J. G. Andrews, "Offloading in heterogeneous networks: Modeling, analysis, and design insights," IEEE Trans. on Wireless Communications, vol. 12, no. 5, pp. 2484-2497, May 2013.

[19] Y. Lin and W. Yu, "Optimizing user association and frequency reuse for heterogeneous network under stochastic model," in Proc. of IEEE Globecom, Atlanta, GA, Dec. 2013.

[20] P. F. Ash and E. D. Bolker, "Generalized Dirichlet tessellations," Geometriae Dedicata, vol. 20, no. 2, pp. 209243, Apr. 1986.

[21] J.-S. Ferenc and Z. Neda, "On the size distribution of Poisson Voronoi cells," Physica A: Statistical Mechanics and its Applications, vol. 385, no. 2, pp. 518-526, Nov. 2007. 